\title{
COVID-19 outbreak in Italy: experimental chest X-ray scoring system for quantifying and monitoring disease progression
}

\author{
Andrea Borghesi ${ }^{1}\left[\right.$ [ $\cdot$ Roberto Maroldi $^{1}$
}

Received: 8 March 2020 / Accepted: 13 April 2020 / Published online: 1 May 2020

(c) Italian Society of Medical Radiology 2020

\begin{abstract}
Severe Acute Respiratory Syndrome Coronavirus 2 (SARS-CoV-2) is a new virus recently isolated from humans. SARS$\mathrm{CoV}-2$ was discovered to be the pathogen responsible for a cluster of pneumonia cases associated with severe respiratory disease that occurred in December 2019 in China. This novel pulmonary infection, formally called Coronavirus Disease 2019 (COVID-19), has spread rapidly in China and beyond. On 8 March 2020, the number of Italians with SARS-CoV-2 infection was 7375 with a $48 \%$ hospitalization rate. At present, chest-computed tomography imaging is considered the most effective method for the detection of lung abnormalities in early-stage disease and quantitative assessment of severity and progression of COVID-19 pneumonia. Although chest X-ray (CXR) is considered not sensitive for the detection of pulmonary involvement in the early stage of the disease, we believe that, in the current emergency setting, CXR can be a useful diagnostic tool for monitoring the rapid progression of lung abnormalities in infected patients, particularly in intensive care units. In this short communication, we present our experimental CXR scoring system that we are applying to hospitalized patients with COVID-19 pneumonia to quantify and monitor the severity and progression of this new infectious disease. We also present the results of our preliminary validation study on a sample of 100 hospitalized patients with SARS-CoV-2 infection for whom the final outcome (recovery or death) was available.
\end{abstract}

Keywords SARS-CoV-2 $\cdot$ COVID-19 $\cdot$ Chest X-ray $\cdot$ Computed tomography $\cdot$ Scoring system

\section{Introduction}

Severe Acute Respiratory Syndrome Coronavirus 2 (SARS$\mathrm{CoV}-2$ ) is a new virus recently isolated from humans. This new virus is a betacoronavirus that belongs to the orthocoronavirinae subfamily of the coronaviridae family [1,2]. SARS-CoV-2 was discovered to be the pathogen responsible for a cluster of pneumonia cases associated with severe respiratory disease that occurred in December 2019 in Wuhan, China [1]. This novel pulmonary infection, formally called Coronavirus Disease 2019 (COVID-19) [3], has spread rapidly throughout China and beyond $[2,4]$.

On 21 February 2020, SARS-CoV-2 infection was also detected in Northern Italy. Since then, the number of

Andrea Borghesi

andrea.borghesi@unibs.it

1 Department of Medical and Surgical Specialties, Radiological Sciences and Public Health, University of Brescia, ASST Spedali Civili of Brescia, Piazzale Spedali Civili, 1, 25123 Brescia, Italy
SARS-CoV-2 infection cases has grown exponentially. On 8 March 2020, the number of Italians with SARS-CoV-2 infection was 7375 with a $48 \%$ hospitalization rate (18\% in intensive care units) and a 5\% mortality rate.

At present, the reference standard to make a definitive diagnosis of SARS-CoV-2 infection is the reverse-transcription-polymerase-chain-reaction assay (RT-PCR) [5].

Radiological imaging plays a crucial role in the detection and management of COVID-19 patients. Computed tomography (CT) imaging is considered the most effective method for the detection of lung abnormalities, particularly in the early stage of the disease [4, 6-12]. Moreover, serial chest $\mathrm{CT}$ imaging with different time intervals (from three to seven days) is also effective in assessing the disease progression (from the time of initial diagnosis of COVID-19 until patient discharge) $[6,7,13]$.

However, the increasing number of hospitalized patients and the consequent increase in radiological examinations would make the constant use of chest CT scan (from diagnosis to discharge) difficult to sustain over time. Therefore, in our Radiology department, we are trying to limit the 
use of CT imaging for monitoring patients with confirmed infection.

Although chest X-ray (CXR) is considered not sensitive for the detection of pulmonary involvement in early-stage disease $[4,14]$, we believe that, in the current emergency setting, CXR (standard or bedside) can be a useful diagnostic tool for monitoring ("day after day") the rapid progression of lung abnormalities in COVID-19, particularly in critical patients admitted to intensive care units.

The radiological quantification of the severity and progression of lung abnormalities is of great importance in determining the appropriate clinical management and respiratory support for infected patients. At the present time, different CT scoring systems and only one CXR scoring system were applied to quantify the pulmonary involvement in COVID-19 [6, 7, 11]. This CXR scoring system is a simple five-point grading tool that was proposed in 2015, and it was designed for non-radiologist clinicians [15]. The goal of this scoring system was to facilitate the clinical grading of CXR reports into five different severity categories in hospitalized patients with acute respiratory infection.

To the best of our knowledge, there are no published papers in which a dedicated CXR grading system for COVID-19 pneumonia has been designed for radiologists.

The aim of this short communication is to present our experimental CXR scoring system that we are applying to hospitalized patients with COVID-19 pneumonia. We also assessed the validity of this CXR scoring system on a sample of 100 hospitalized patients with SARS-CoV-2 infection for whom the final outcome (recovery or death) was available.

\section{Materials and methods}

\section{CXR scoring system}

Based on the current knowledge of common chest CT findings in COVID-19 pneumonia (ground-glass opacity with or without patchy consolidation) $[4,6-8,10-12]$, we designed a dedicated CXR scoring system for hospitalized patients with SARS-CoV-2 infection (confirmed by RT-PCR).

Our CXR scoring system for COVID-19 pneumonia (which we named Brixia score) includes two steps of image analysis.

In the first step, the lungs are divided into six zones on frontal chest projection (posteroanterior or anteroposterior projection according to the patient position) (Fig. 1):

- Upper zones (A and $D$ ): above the inferior wall of the aortic arch

- Middle zones ( $B$ and $E$ ): below the inferior wall of the aortic arch and above the inferior wall of the right inferior pulmonary vein (i.e., the hilar structures)

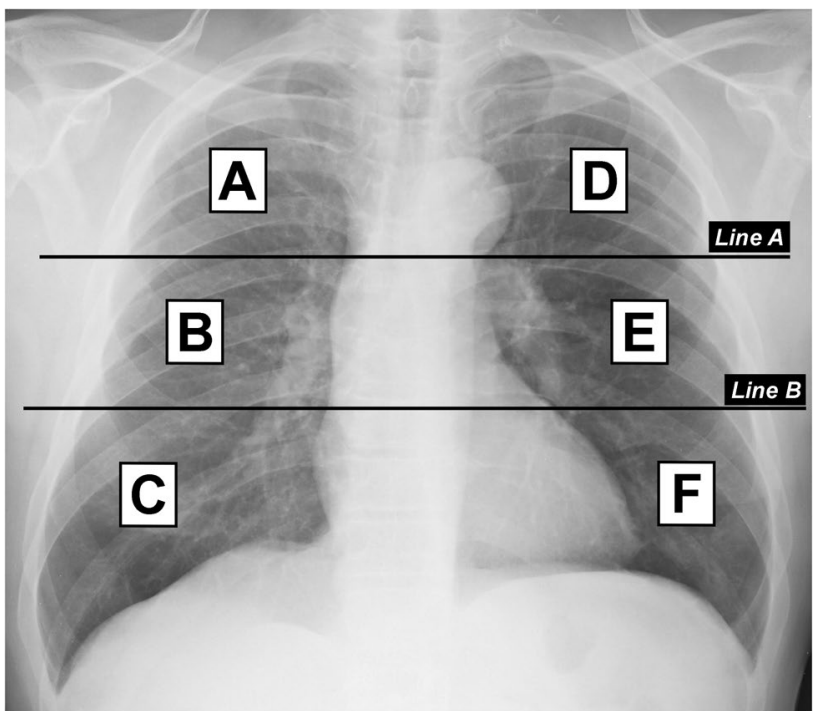

Fig. 1 Division of lungs into six zones on frontal chest radiograph. Line $A$ is drawn at the level of the inferior wall of the aortic arch. Line $B$ is drawn at the level of the inferior wall of the right inferior pulmonary vein. $A$ and $D$ upper zones; $B$ and $E$ middle zones; $C$ and $F$ lower zones

- Lower zones $(C$ and $F$ ): below the inferior wall of the right inferior pulmonary vein (i.e., the lung bases)

For technical reasons (for example bedside CXR in critical patients), it could be difficult to identify some anatomical landmarks. In such cases, we recommend dividing each lung into three equal zones.

In the second step, a score (from 0 to 3 ) is assigned to each zone based on the lung abnormalities detected on frontal chest projection as follows (Fig. 2):

- Score 0 no lung abnormalities

- Score 1 interstitial infiltrates

- Score 2 interstitial and alveolar infiltrates (interstitial predominance)

- Score 3 interstitial and alveolar infiltrates (alveolar predominance)

The scores of the six lung zones are then added to obtain an overall "CXR SCORE" ranging from 0 to 18 .

The "CXR SCORE" is entered at the end of the descriptive report. Near to the overall score, the partial score of each zone (from $A$ to $F$ ) is also entered between square brackets.

Other CXR findings (such as pleural effusion, pulmonary vessel enlargement), not included in the scoring system, are recorded in the descriptive part of the CXR report.

An example of our CXR report is shown below:

Chest x-ray

Symmetrical lung expansion. 


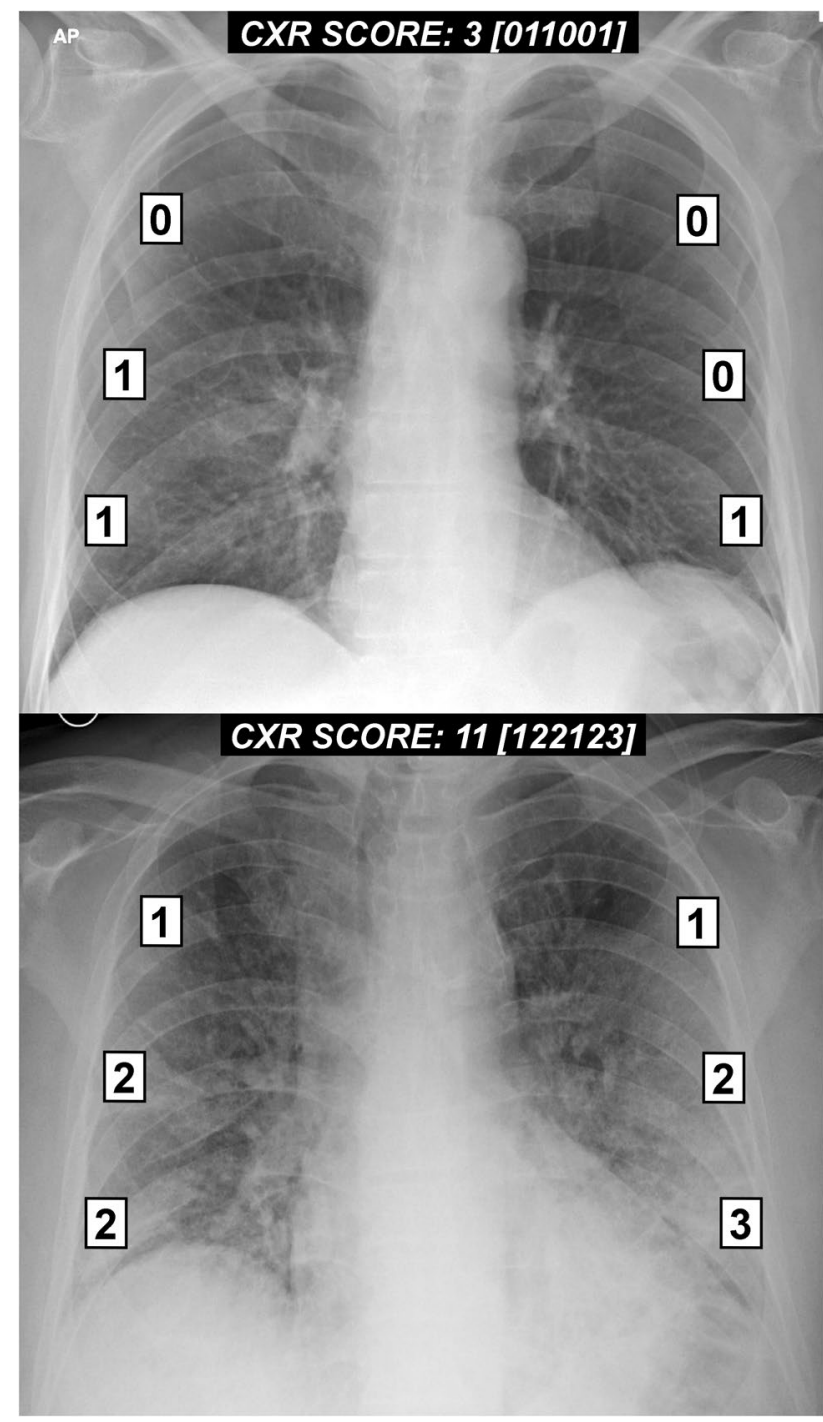

Fig. 2 Examples of the chest X-ray scoring system in two patients with COVID-19 pneumonia

Interstitial and alveolar infiltrates in the middle and lower zones of both lungs, greater to the left side.

No pleural effusion.

Mediastinum and heart size within normal limits.

CXR SCORE: 10 [022033]

\section{Validation study}

To assess the validity of our CXR scoring system, we selected 100 hospitalized patients with SARS-CoV-2 infection for whom the final outcome (recovery or death) was available. The selected sample was obtained from our departmental digital archive. All CXR reports containing the new scoring system were retrieved. For each patient, only the CXR report with the highest score was considered for this validation study. The frontal chest projection linked to these $100 \mathrm{CXR}$ reports was independently assessed by an experienced thoracic radiologist who reassigned the score for each CXR examination.

To determine the agreement between radiologists in the application of the new CXR scoring system, the first score of each CXR examination was compared with the second score reassigned by the experienced thoracic radiologist. To assess this agreement, the weighted kappa $\left(\mathrm{k}_{\mathrm{w}}\right)$ and $95 \%$ confidence interval (CI) were calculated. We use the $\mathrm{k}_{\mathrm{w}}$ (specifically the linear $\mathrm{k}_{\mathrm{w}}$ ) because the CXR scoring system was designed on an 18-point continuous-ordinal scale and the degree of disagreement between the scores had a different weight. The Mann-Whitney U-test was also used to compare CXR scores with the final outcome (recovery or death) in selected patients. We used this non-parametric test because the CXR score was not normally distributed. All statistical analyses were performed using commercial software (MedCalc Statistical Software version 19, Ostend, Belgium). $P$-values of less than 0.05 were considered statistically significant.

\section{Results}

The score entered in the CXR reports ranged from 0 to 16 (median 6.5; interquartile range 2-11). The CXR score reassigned by the thoracic radiologist ranged from 0 to 15 (median, 7; interquartile range, 3-10). The CXR scoring agreement was very good $\left(\mathrm{k}_{\mathrm{w}}, 0.82 ; 95 \% \mathrm{CI}, 0.79-0.86\right)$.

For both semi-quantitative analyses (retrieved from reports, and performed by the thoracic radiologist), the CXR score was significantly higher in patients who died than those discharged from the hospital $(p \leq 0.002)$.

\section{Discussion}

In this short communication, we presented our original CXR scoring system for grading the severity of COVID-19 pneumonia. This scoring system is designed exclusively for a semi-quantitative assessment of the severity and progression of pulmonary involvement in hospitalized patients with COVID-19 (Fig. 3). It is quite simple and can be easily replicated in other clinical settings.

We obviously realize that this method required further studies to confirm its validity because the score depends mainly on the quality of the CXR images and the experience of the observers. However, in the preliminary validation study, we found that the inter-observer agreement was very good and the CXR score was a useful parameter for predicting mortality in hospitalized patients with SARSCoV-2 infection.

In conclusion, we consider this scoring tool to be very promising due to its ability to provide, in a very clear and 


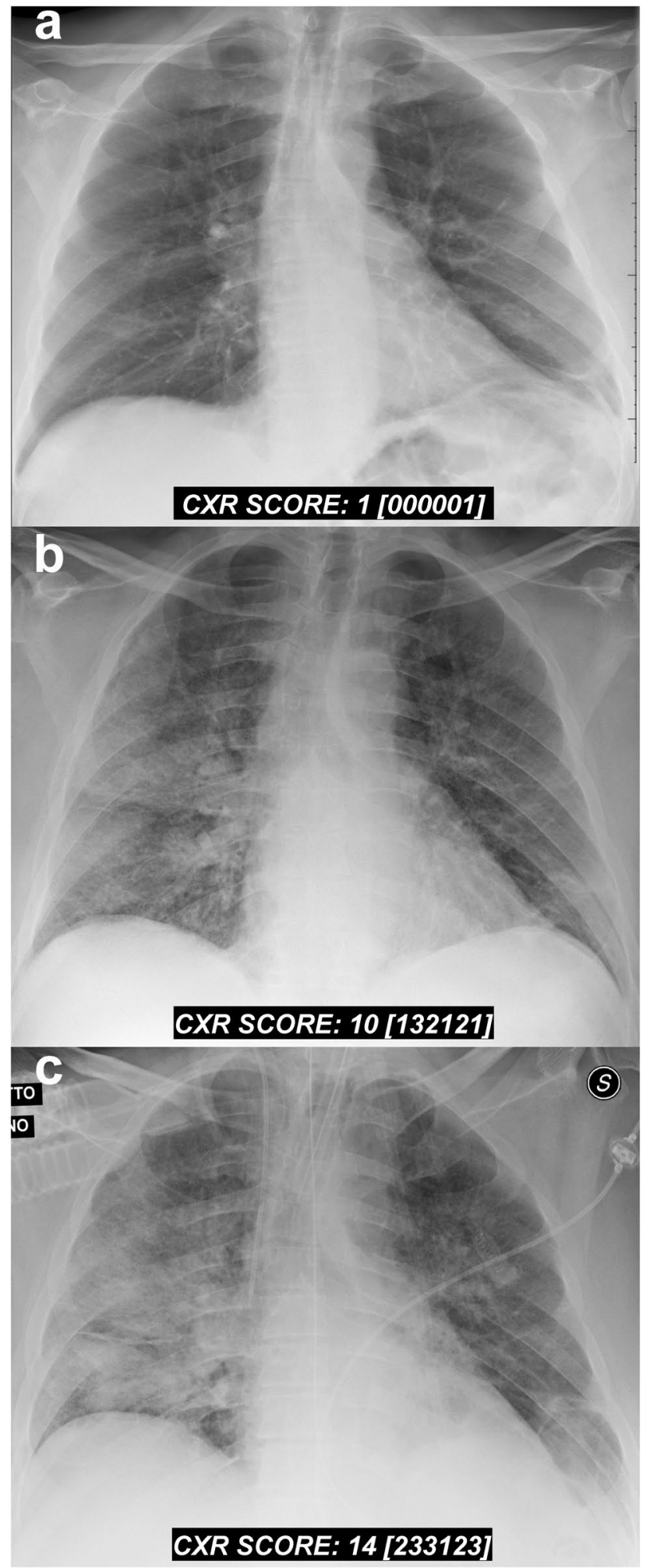

Fig. 3 Serial chest X-ray findings in a 72-year-old male patient with COVID-19 pneumonia. a Baseline frontal chest radiograph performed on the day of admission (one day after the onset of fever). A rapid progression of the lung disease is shown on radiographic follow-ups performed at day 4 (b) and day 5 (c) post-hospitalization straightforward way, relevant information for clinicians by enhancing the role of radiologists in this long and tiring battle against this new viral pneumonia.

Funding The author states that this work has not received any funding.

\section{Compliance with ethical standards}

Conflict of interest The authors declare that they have no conflict of interest.

Ethical standards All procedures followed were in accordance with the ethical standards of the responsible committee on human experimentation (institutional and national) and with the Helsinki Declaration of 1975, as revised in 2008 .

\section{References}

1. Zhu N, Zhang D, Wang W et al (2020) A novel coronavirus from patients with pneumonia in China, 2019. N Engl J Med. https:// doi.org/10.1056/nejmoa2001017

2. Sun P, Lu X, Xu C, Sun W, Pan B (2020) Understanding of COVID-19 based on current evidence. J Med Virol. https://doi. org/10.1002/jmv.25722

3. World Health Organization (2020) WHO Director-General's remarks at the media briefing on 2019-nCoV. https://www.who. $\mathrm{int} / \mathrm{dg} / \mathrm{speeches/detail/who-director-general-s-remarks-at-the-}$ media-briefing-on-2019-ncov-on-11-february-2020

4. Zu ZY, Jiang MD, Xu PP, Chen W, Ni QQ, Lu GM, Zhang LJ (2020) Coronavirus disease 2019 (COVID-19): a perspective from China. Radiology. https://doi.org/10.1148/radiol.2020200490

5. Chinese Society of Radiology (2020) Radiological diagnosis of new coronavirus infected pneumonitis: expert recommendation from the Chinese Society of Radiology (First edition). Chin J Radiol 54:E001-E001

6. Bernheim A, Mei X, Huang M et al (2020) Chest CT findings in coronavirus disease-19 (COVID-19): relationship to duration of infection. Radiology. https://doi.org/10.1148/radiol.2020200463

7. Pan F, Ye T, Sun P et al (2020) Time course of lung changes on chest CT during recovery from 2019 novel coronavirus (COVID19) pneumonia. Radiology. https://doi.org/10.1148/radiol.20202 00370

8. Shi H, Han X, Jiang N et al (2020) Radiological findings from 81 patients with COVID-19 pneumonia in Wuhan, China: a descriptive study. Lancet Infect Dis 3099:1-10. https://doi.org/10.1016/ S1473-3099(20)30086-4

9. Fang Y, Zhang H, Xie J, Lin M, Ying L, Pang P, Ji W (2020) Sensitivity of chest CT for COVID-19: comparison to RT-PCR. Radiology. https://doi.org/10.1148/radiol.2020200432

10. Kim H (2020) Outbreak of novel coronavirus (COVID-19): what is the role of radiologist? Eur Radiol. https://doi.org/10.1007/ s00330-020-06748-2

11. Yoon SH, Lee KH, Kim JY et al (2020) Chest radiographic and CT findings of the 2019 novel coronavirus disease (COVID-19): analysis of nine patients treated in Korea. Korean J Radiol. https ://doi.org/10.3348/kjr.2020.0132

12. Li Y, Xia L (2020) Coronavirus disease 2019 (COVID-19): role of chest CT in diagnosis and management. AJR Am J Roentgenol 215:1-7. https://doi.org/10.2214/AJR.20.22954 
13. Wei J, Xu H, Xiong J et al (2020) 2019 novel coronavirus (COVID-19) pneumonia: serial computed tomography findings. Korean J Radiol. https://doi.org/10.3348/kjr.2020.0112

14. Ng M-Y, Lee EY, Yang J et al (2020) Imaging profile of the COVID-19 infection: radiologic findings and literature review. Radiol Cardiothorac Imaging 2:e200034. https://doi.org/10.1148/ ryct.2020200034

15. Taylor E, Haven K, Reed P et al (2015) A chest radiograph scoring system in patients with severe acute respiratory infection: a validation study. BMC Med Imaging 15:1-10. https://doi. org/10.1186/s12880-015-0103-y

Publisher's Note Springer Nature remains neutral with regard to jurisdictional claims in published maps and institutional affiliations. 